\title{
Can we Prevent Morbidities and Mortalities of Diabetes
}

\section{Mellitus?}

\begin{abstract}
Hussain HY*
Hamid Yahya Hussain, Primary Health Care Service Sector, Dubai Health Authority, Dubai
\end{abstract}

*Corresponding author: Hamid Yahya Hussain, Primary Health Care Service

Sector, Dubai Health Authority, Dubai, Email: hyHussain@dha.gov.ae

\author{
Editorial \\ Volume 2 Issue 2
}

Received Date: August 05, 2018

Published Date: August 17, 2018

\section{Editorial}

Diabetes Mellitus stayed since when, as one of the major contributes of morbidities such as of blindness, end-stage renal failure, non-traumatic limb amputations, and cardiovascular morbidity, and stroke. More to that, the diseases kept being recognized as a leading cause of mortalities all the way through with all sequel implications regarding the quality of life and coast burdens. Thus Prevention and management of diabetes and its complications will stand as the golden standard strategy and best long-term investment of high returns. Recently generated pieces of evidence by international literature revealed that effective approaching of Diabetes always encountering challenges, three fronts has been identified to tackling diabetes: Primary prevention, secondary prevention (of diabetes complications), and tertiary prevention (of morbidity and mortality from established complications). Abundant evidence now clearly available, that type 2 diabetes, which accounts for the vast majority of prevalence, for greater than $90 \%$ of diabetes worldwide, is preventable. The main bulk of patients with diabetes receive routine care from primary care providers are not treated by endocrinologists. Primary care setting professionals e.g. Internists, family practice physicians, and physician extenders with advanced skills, are carrying out the main tasks of implementing standards of care assigned for persons with diabetes. Accumulating evidence revealed that the complications of diabetes are preventable by implementing a tight policy of glycemic control and effective risk reduction. Even though the complications have set in, strict glucose control process, dramatically declining the risk of complications progressions. Thus, strategies that enable translation of existing scientific data to pragmatic benefits can be properly addressed. There are many competent strategies can be applied in diabetes prevention and control settings, can lead to tremendous reduction of diabetes-related morbidities and eventually mortalities at primary health care level, as well as other healthcare levels , likewise, surveillance for microvascular complications; efficient use of medications provider education; goal setting; ; patient empowerment through promotion of lifestyle and self-care practices cardiovascular risk reduction and stratification of patients and triaging of those with poor glycemic control for more intensive management.

Improving health outcomes of Diabetic patients has been identified as significantly associated with behavioral related approach including:

Patients related behaviors can be done through Selfmonitoring of blood glucose (SMBG) for all persons with type 1 diabetes and for patients with insulin-treated type 2 diabetes. SMBG proved to be correlated with improved health outcomes for persons with type 1 diabetes as shown by clinical trials. Calorie and fat intake reduction, associated with improved glycemic control as well as with weight control. Cardiovascular protection can be offered by aspirin use for persons with and without diabetes especially for persons with diabetes who are aged $>30$ years and who have no contraindications. Improved glycemic control among persons with diabetes proved to be associated with practicing physical activities. And the effect of smoking on morbidity and mortality from micro- and macro vascular complications associated with diabetes.

Behaviors related to Providers: glycosylated hemoglobin (GHb), lipids, foot lesions, and neuropathy which are monitoring by providers, has been remarkably linked to improving. Routine annual screening for retinopathy and nephropathy, and 


\section{Epidemiology international journal}

identifying abnormalities, associated with improved health outcomes among persons with diabetes.

Long and Short-Terms Outcomes. Long-term health outcomes among persons with diabetes. Significantly associated with, glycemic control, blood pressure, lipid levels, proteinuria and renal function, weight, and the presence of foot lesions are all associated. 